\title{
Anti-CD40L Fc-Fusion Protein BMS-986004
}

National Cancer Institute

\section{Source}

National Cancer Institute. Anti-CD40L FC-Fusion Protein BMS-986004. NCI Thesaurus. Code C120140.

A dimeric fusion protein composed of the C-terminus of the domain antibody $(\mathrm{dAb})$ BMS2h-572-633 targeting the CD40 ligand (CD40L or CD154) linked to a modified Fc fragment of immunoglobulin G1 (IgG1), with potential immunomodulatory activity. Upon intravenous administration, the peptide moiety of anti-CD40L antibody BMS-986004 specifically targets and binds to CD40L expressed on T-lymphocytes. This prevents the binding of CD40L to its cognate receptor CD40 expressed on B-lymphocytes, macrophages, and dendritic cells (DCs). This prevents T-cell mediated proliferation and differentiation of B-cells, and prevents the production of antibodies. By inhibiting both the production of anti-glycoprotein (GP) IIb/IIIa antibodies by B-cells and GPIIb/IIIadependent T-cell proliferation, BMS-986004 may prevent platelet destruction and may increase platelet counts in idiopathic thrombocytopenic purpura (ITP). The direct binding of BMS-986004 to CD40L on platelets further prevents CD40L/CD40-mediated destruction by macrophages and DCS in ITP. The modified Fc domain prevents the binding of BMS-986004 to the Fc receptor FcgammaRIIA on platelets, thereby preventing FcgammaRIIA-dependent platelet activation and anti-CD40L-induced thromboembolism. CD40L, a transmembrane protein of the tumor necrosis factor (TNF) superfamily, is primarily expressed on activated T-cells, but is also expressed on eosinophils, basophils, natural killer (NK) cells, mast cells, platelets and activated endothelial cells. 